\title{
Design and Development of Virtual Objects to be Used with Haptic Device for Motor Rehabilitation
}

\author{
Jatin B. Maniya, Pushparaj Mani Pathak, Bhanu Kumar Mishra \\ Mechanical and Industrial Engineering Department, Indian Institute of Technology, Roorkee, India. \\ Email: pushpfme@iitr.ernet.in
}

Received August $2^{\text {nd }}, 2010$; revised August 25 ${ }^{\text {th }}, 2010$; accepted September $2^{\text {nd }}, 2010$.

\begin{abstract}
This paper presents design and development of virtual objects to be used with haptic device PHANTOM Omni for motor rehabilitation by incorporating visual and haptic feedback. Developed predominantly for augmentation of motor skill of patient, the objects could also be used for teaching and enhancing writing skill of children. The virtual objects include English alphabets, numbers, string and mazes, and games. The scheme has been evaluated on children from 11 to 14 years age. The experiment gives good results.
\end{abstract}

Keywords: Haptic, Motor Rehabilitation, Virtual Environment

\section{Introduction}

Haptic is derived from the Greek verb "haptesthai" meaning "to touch" and it refers to the science of touch and force feedback in human-computer interaction. As haptic devices are evolving and getting cheaper and more compact in size options are explored by the researchers for their use in medical applications. Haptic device provides an excellent addition to virtual environment due to the force feedback and tactile input/output when it is used for rehabilitation of patients suffering with motor disorder. This property also helps the children who are learning to write, who otherwise get this information from teacher's hand when he/she holds their hand.

Generally, stroke sufferer loses the skill of writing and they are unable to write again. From literature it is observed that little work has been carried out on enhancing writing skill of patient recovering after stroke. Many stroke victim state loss of handwriting ability as one of their biggest disabilities compared to other injury [1]. In 1998, Prisco, Avizzano, Calcara, Ciancio, Pinna and Bergamasco [2] have presented an immersive Virtual Environment (VE) including visual, auditory and haptic feedbacks, which had been designed specifically to help in recovery or improve the motor dexterity of the arm and hand of patients affected by motor disorder. They created kitchen base environment in which subject has to execute task like burning stove, putting coffee pot on stove etc. and they got satisfactory result when tried on patient. During the same time Henmi and Yoshikawa [3] developed virtual calligraphy system to transfer skill of teacher to student. They concluded that the student's position and force trajectories resemble closer to those of the teacher's as the exercise advances. Gillespie, O’Modhrain, Tang, Zaretzky and Pham [4] developed virtual environment, which can demonstrate certain object manipulation techniques. Although average performance time for the experimental groups did not improve faster than that of the control group, the optimal strategy was successfully communicated to the experimental groups. Pernalete, Yu, Dubey and Moreno [5] has developed an assessment and training procedure that result in improving handwriting taking advantage of the force feedback provided by the haptic device, incorporating inertia and viscosity effects to decrease the tremor in the hand as well as to stimulate the muscles. Teo, Burdet and Lim [6] introduced a virtual teaching system for Chinese ideograms that guides movement by haptic and visual feedback. It resulted in improvement of performance, in particular for beginners learning Chinese writing with a pen. Learning was evaluated by considering accuracy and speed of writing. Feygin, Keehner and Tendick [7] investigated use of haptics for skills training. Their study indicated that haptic guidance is effective in training. The performance was improved in terms of position, shape, timing, and drift. In 2005, Pemalete et al. [8] performed a test on a force-reflecting haptic interface device, PHANTOM with the GHOST SDK Software. 
They used these devices to improve the hand eye coordination problem of children. In 2005, Mullins, Mawson and Nahavandi [9] have developed a method of controlling a user's hand through the process of writing. They introduced Proportional Differential (PD) controller that decreases the overshoot and reduces settling time to acceptable boundaries in PHANTOM. Grantner, Gottipati, Pernalete, Fodor and Edwards [10] have developed an intelligent decision support system for eye-hand coordination assessment which is based on fuzzy logic algorithm. It takes decision on whether subject should be allowed to move to next stage or not based on his/her previous performance. In 2007, Mansour, Eid, and Saddik [11] presented a multimedia system designed to facilitate learning of alphabetical handwriting of various languages by incorporating visual, auditory, and haptic feedback. Gouy-Pailler, Zijp-Rouzier, Vidal and Chêne [12] have represented a system consisted of a haptic pen and computer generated virtual environment. This system was used to teach the children, especially visually impaired children. This system was specially designed to teach geometric lessons. Subjects were asked to regenerate the shapes immediately after training. Experiments performed on them have shown impressive results, specifically in case of totally blind subjects. Palluel-Germain et al. [13] have developed a system incorporating a visuo-haptic device 'Telemaque' to increase the fluency of handwriting production of cursive letters in kindergarten children i.e., before formal handwriting learning begins. Rassmus-Grohn, Magnusson and Efiring [14] presented the evaluation of an audio-haptic editor and explorer for virtual 2D relief drawings that allow visually impaired users to create and explore graphical images. In 2007, Kayyali, Shirmohammadi, Saddik and Lemaire [15] presented a system that uses haptics, in conjunction with virtual environments, to provide a rich media environment for motor rehabilitation of stroke patients. The system also provides occupational therapists with a human-computer interface that allows for easier set-up, more facilitated interaction with the system, and provides a more autonomous means for the progression of the patient. The system had been modified, after thorough analysis by a group of experienced occupational therapists. In [16], Bayart, Pocheville and Kheddar have presented a progressive guiding system designed as a software module for the framework I-TOUCH using simple XML code. They have built features like fully, partially and no guidance from haptic device, PHANTOM Omni. Then from the experiments carried out over three groups, they concluded that the one group given partial haptic help, perform well compared to other two.

This work presents virtual environment where objects, numbers, words and games have been created in open platform. The earlier work reported has been related with Chinese character [6]. Here we have created virtual objects that comprises of complete English alphabets, English numerals from 0 to 9, English words, and games. These virtual objects are interfaced with SensAble PHANTOM Omni haptic device. Presented system can provide training to enhance writing skill of motor stroke patients and children. This method of haptic training has the potential to stimulate re-learning in the brain of stroke victim. Alphabets, numbers and full words have been created with aim of increasing the learning ability whereas maze has been created to improve coordination skill in subjects. A ball tracking game has been created with aim of increasing the concentration of subjects. These objects have been created based on a study carried out on motor disordered patients. To validate the idea experiments have been carried out on subjects aged 11-14 years. The experimental results clearly indicate improvement in the learning ability of the subjects. The principal contribution of this work is logical creation of virtual objects which have been linked with PHANTOM Omni haptic device and testing of these objects on subjects for evaluating learning level.

\section{Brief Description of Haptic PHANTOM Omni}

The PHANTOM Omni model is the most cost-effective haptic device available today. Three degrees of force, in the $x, y$ and $z$, direction are achieved through motors that apply torque at each joint in the robotic arm. The position and orientation of the pen are tracked through encoders in the robotic arm. Figure 1 shows setup for rehabilitation.

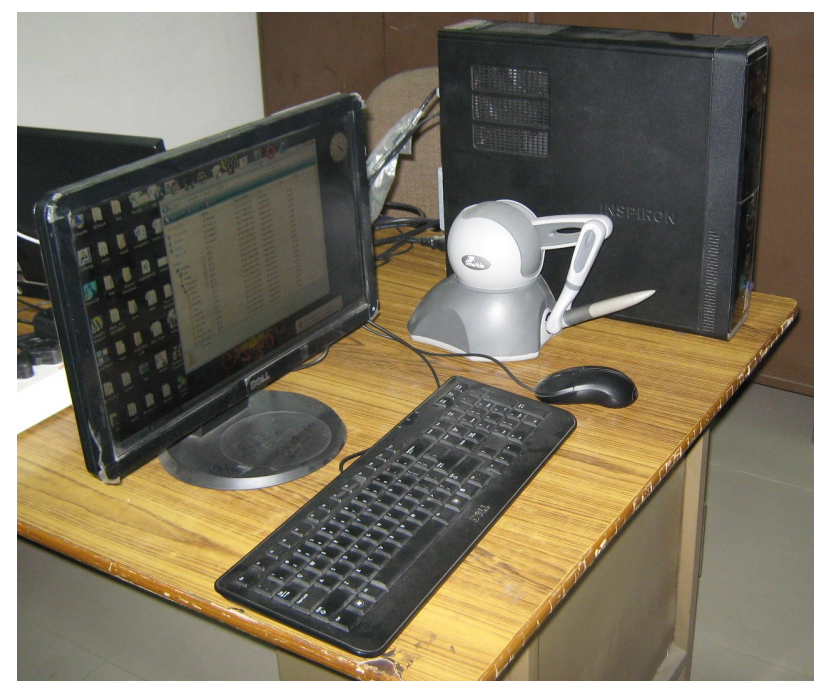

Figure 1. Haptic rehabilitation setup with PHANTOM Omni and computer. 


\section{Result of Study on Motor Disordered Subjects}

Some subjects were studied to find major difficulties faced by them. This information was used to create virtual environment that can be used for augmentation of their motor skills. Table 1 shows the details of the subjects, difficulties faced by them and the reason for the difficulty. Conclusion from Table $\mathbf{1}$ is that motor disordered patients suffer from uncontrolled hand movement, due to which they cannot write properly.

\section{Design of Virtual Objects for Training}

The system consists of alphabets, numbers, string, maze and game. Presented system has been designed to give partial guidance to the subject. This system has different exercises on alphabets and numbers which are partially guided. Here partially guided means object will not react until subject is moving his hand within range of pre-specified path. When it tries to move outside of object, system will exert force on stylus and will keep it in range only. Thus, the system is neither fully guided nor fully free. To attain this feature in letters, we created 3-D model of letters which consists of walls.

In present system, Open Graphic Library Utility Toolkit (GLUT) is used for graphical presentation on screen. Haptic Device Application Programming Interface (HDAPI) provides low-level access to the haptic device. It also enables haptics programmers to render forces directly, offer control over configuration, the runtime behavior of the drivers. Haptic Library Application Programming Interface (HLAPI) provides high-level haptic rendering and allows significant reuse of existing OpenGL code and greatly simplifies synchronization of the haptics and graphics threads.

Table 1. Difficulties faced by disordered subjects.

\begin{tabular}{|c|c|c|c|}
\hline S.N. & Subjects & Type of difficulty & Reasons \\
\hline 1 & $\begin{array}{c}\text { Subject A; } \\
\text { Age-14 Years }\end{array}$ & $\begin{array}{l}\text { Judgment about space and } \\
\text { time, poor handwriting, } \\
\text { language impairment }\end{array}$ & $\begin{array}{l}\text { Motor disorder } \\
\text { from childhood }\end{array}$ \\
\hline 2 & $\begin{array}{c}\text { Subject B; } \\
\text { Age-19 Years }\end{array}$ & $\begin{array}{l}\text { Uncontrolled body } \\
\text { movement, could not stand } \\
\text { still }\end{array}$ & $\begin{array}{l}\text { Motor disorder } \\
\text { developed after } \\
\text { teenage }\end{array}$ \\
\hline 3 & $\begin{array}{c}\text { Subject C; } \\
\text { Age-53 Years }\end{array}$ & $\begin{array}{l}\text { Could not perform normal } \\
\text { domestic work, could not } \\
\text { write }\end{array}$ & Stroke victim \\
\hline 4 & $\begin{array}{c}\text { Subject D; } \\
\text { Age-35 Years }\end{array}$ & $\begin{array}{l}\text { Drop things frequently, } \\
\text { awkward gait pattern }\end{array}$ & $\begin{array}{l}\text { Motor disorder } \\
\text { developed after } \\
\text { teenage }\end{array}$ \\
\hline 5 & $\begin{array}{c}\text { Subject E; } \\
\text { Age-70 Years }\end{array}$ & $\begin{array}{l}\text { Problem with fine motor skill } \\
\text { like cutting food, getting cup } \\
\text { of tea, frequent fatigue }\end{array}$ & $\begin{array}{c}\text { Faced motor } \\
\text { disorder after } 65 \\
\text { years }\end{array}$ \\
\hline
\end{tabular}

\subsection{Alphabet and Number System}

With the help of alphabet and number system virtual object, one can learn to write alphabet and numbers. The letters has been created in PRO/ENGINEER (Pro/E), solid modeling software. Using this model along with OpenHaptic Toolkit, we created scenario in which one can feel the shape of particular letter and move the cursor along with it. As the subject moves his hand through the shape, the stylus will not exert any reaction force on subject hand. But when stylus tries to run out of the wall of letter, subject will feel reaction force and it could not move beyond that limit. This ensures that subject hand remains within certain limit and thus the subject motion is neither free nor fully constrained. We also aided visual feedback through picture, which helps subject to remember used letter and correlate that picture to specific word. The flowchart used for creation of a letter is shown in Figure 2. First of all letters or numbers are modeled in Pro/E environment. The created file is in .prt format. This created file is converted in to .3ds format. Then model is imported in visual $\mathrm{C}++$ environment. The properties of object like mass, stiffness, damping, friction etc. are set. Add picture of the object as visual aid. Also spelling of the object is added. The created alphabet $Q$ is shown in Figure 3.

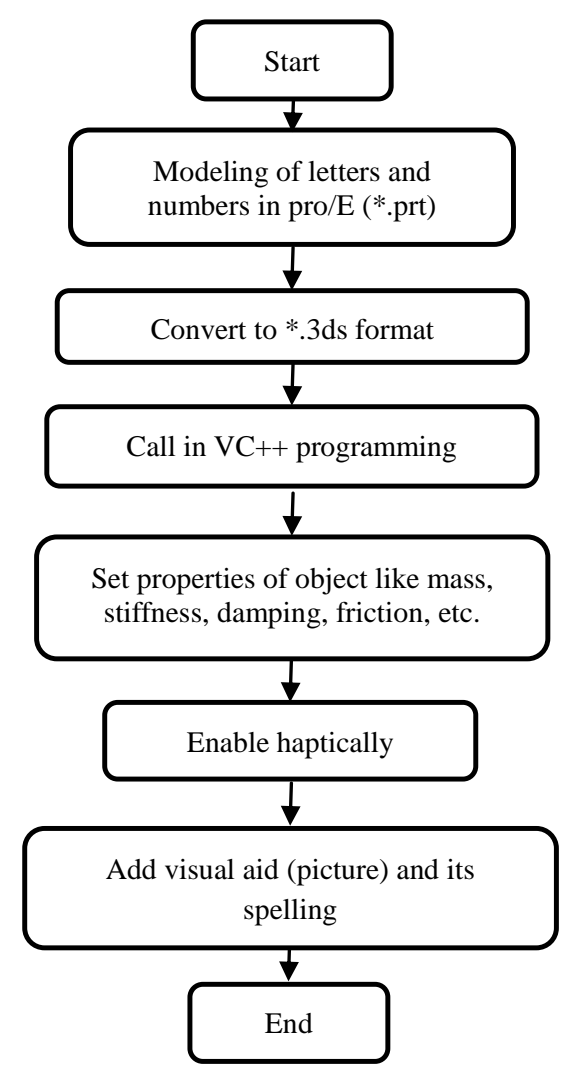

Figure 2. Flowchart for alphabet. 
Creation of numbers is similar to alphabets. Its window contains a number and its spelling printed on a sphere. The snapshot of developed object is shown in Figure 4. The algorithm followed for its construction is similar to that used for alphabets.

\subsection{String}

After one gets practice on letters and number system, the next stage is to learn group of letters i.e. words. So, next set of exercise can be practice on strings. String is constructed using constrained motion scenario. The flowchart used for creation of string is shown in Figure 5. The screen shot of created string is shown in Figure 6.

\subsection{Constrained Wall Maze}

One can practice on constrained maze exercises and can build ability to take decision and follow the path. The created virtual object will not react if subject moves his hand on pre-defined path, but when he/she tries to deviate from it, he/she feel that the motion in that direction is constrained. Thus in this exercise subject could not move his hand in any direction but only pre-specified way. To

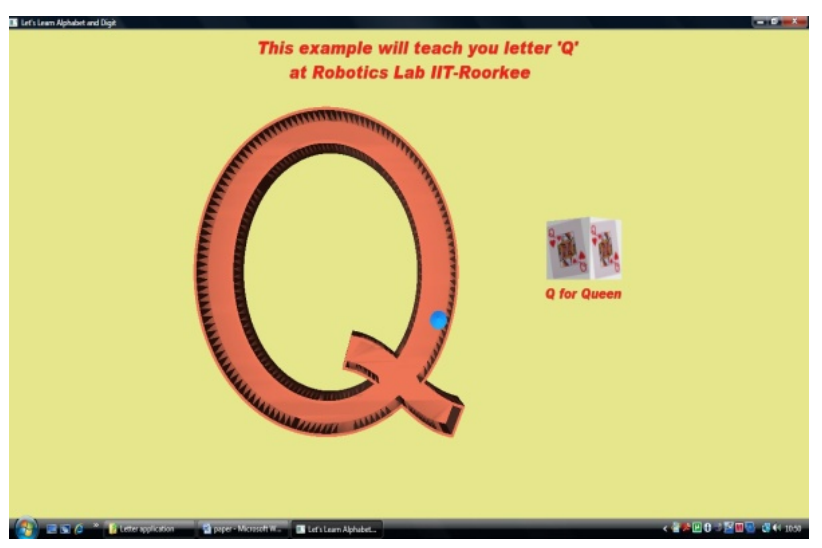

Figure 3. Alphabet exercise.

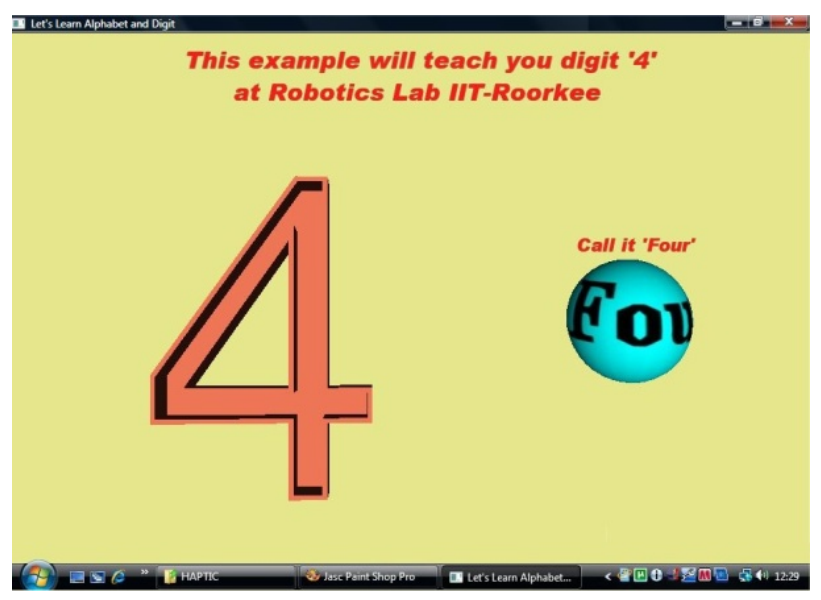

Figure 4. Letter exercise.

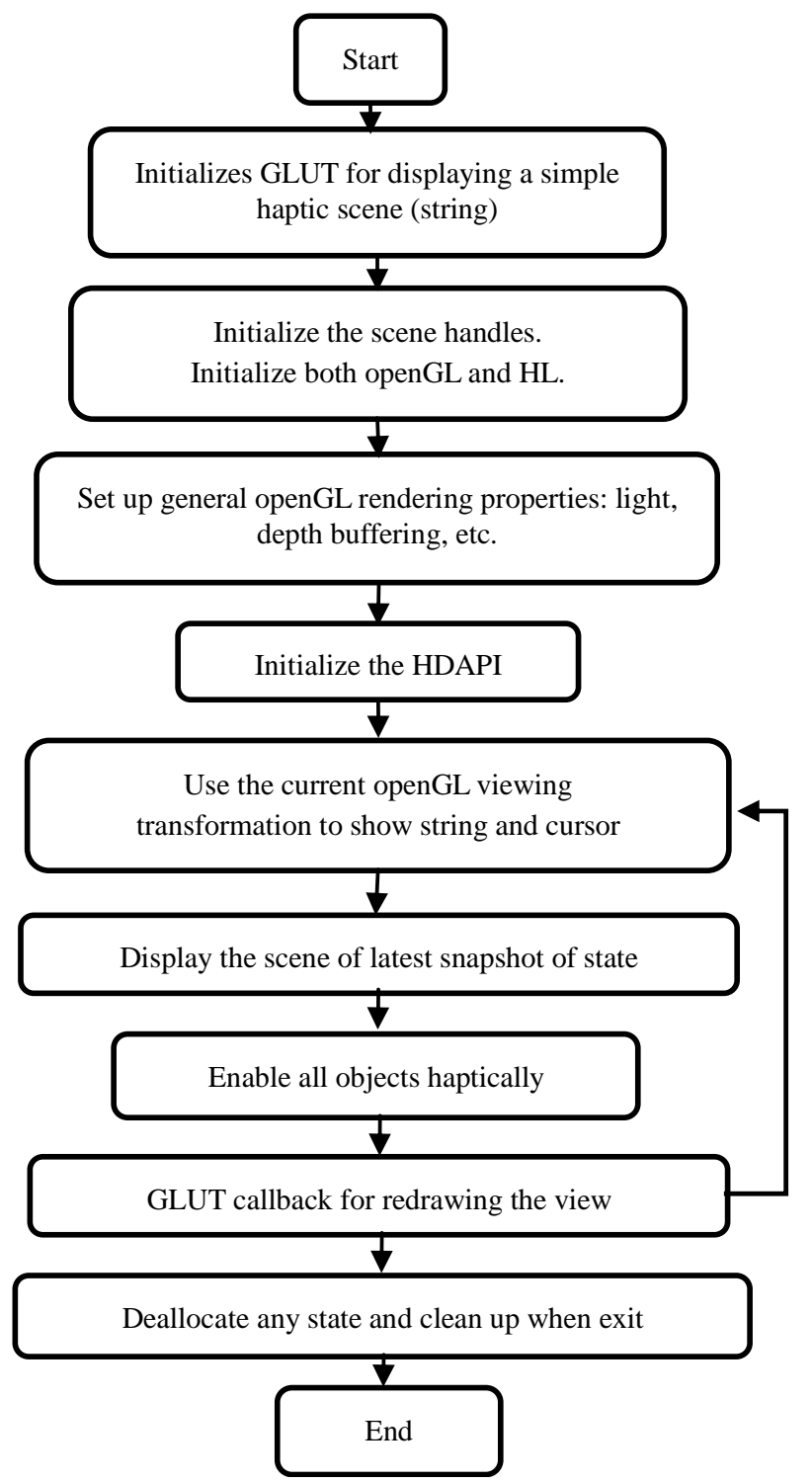

Figure 5. Flowchart for string.

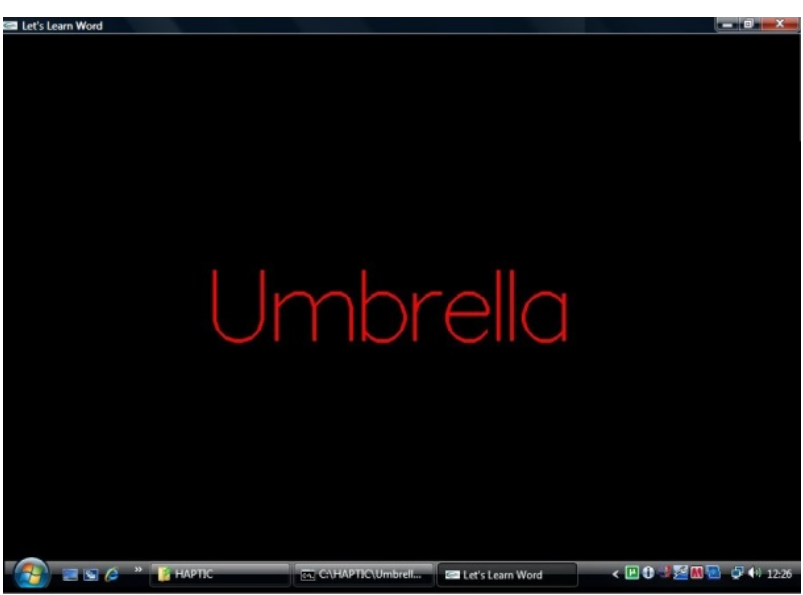

Figure 6. Word (string) exercise. 
reach at finish point from start point, subject has to take many decisions about the path which he has to choose to reach at end point. This exercise will help to augment decision making skill of subject. The flow chart used for creation of maze is similar to that used for string. The created object is shown in Figure 7.

Another object called wall maze is created using basic shape-block. The purpose of this maze system is to compare it with constrained maze and to see which one is more effective. The increase level of difficulty of maze makes it difficult for the subject to reach the end point. Figure 8 shows the flowchart used in creation of the object. Figure 9 shows the snapshot of third level in maze exercise. The difference between maze of Figure $\mathbf{7}$ and $\mathbf{9}$ is that in Figure 7 cursor is guided along the wall of the maze whereas in Figure 9 cursor is guided to move between the walls of the maze.

\subsection{Game}

The virtual objects are created to simulate game like environment. The subjects can be asked to play these games. These will be a good exercise for them. In these exercises, the ball moves on screen randomly and subject has to move his hand along with it to make sure that contact is not lost. As difficulty level increases, the speed of ball increases, so, the chance of lose of contact increases and subject has to take relatively quick decision to keep touching the ball. The snap shot of created game is shown in Figure 10.

\subsection{Graphic User Interface (GUI)}

To access all the exercises from one platform, graphic user interface (GUI) has been developed. Through GUI one can launch any exercise by clicking on desired application. But before launching any application, one must make sure that previous one is closed. This is because thread used for previous application must be de-allocated

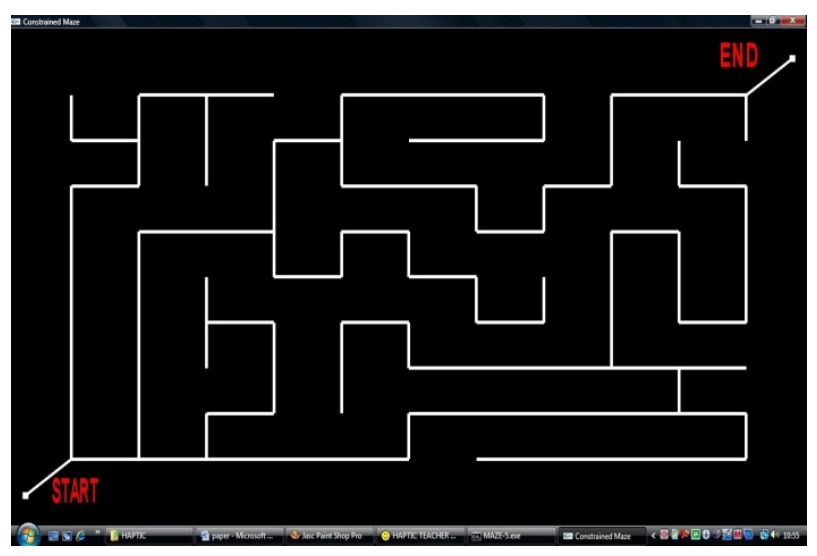

Figure 7. Constrained maze exercise.

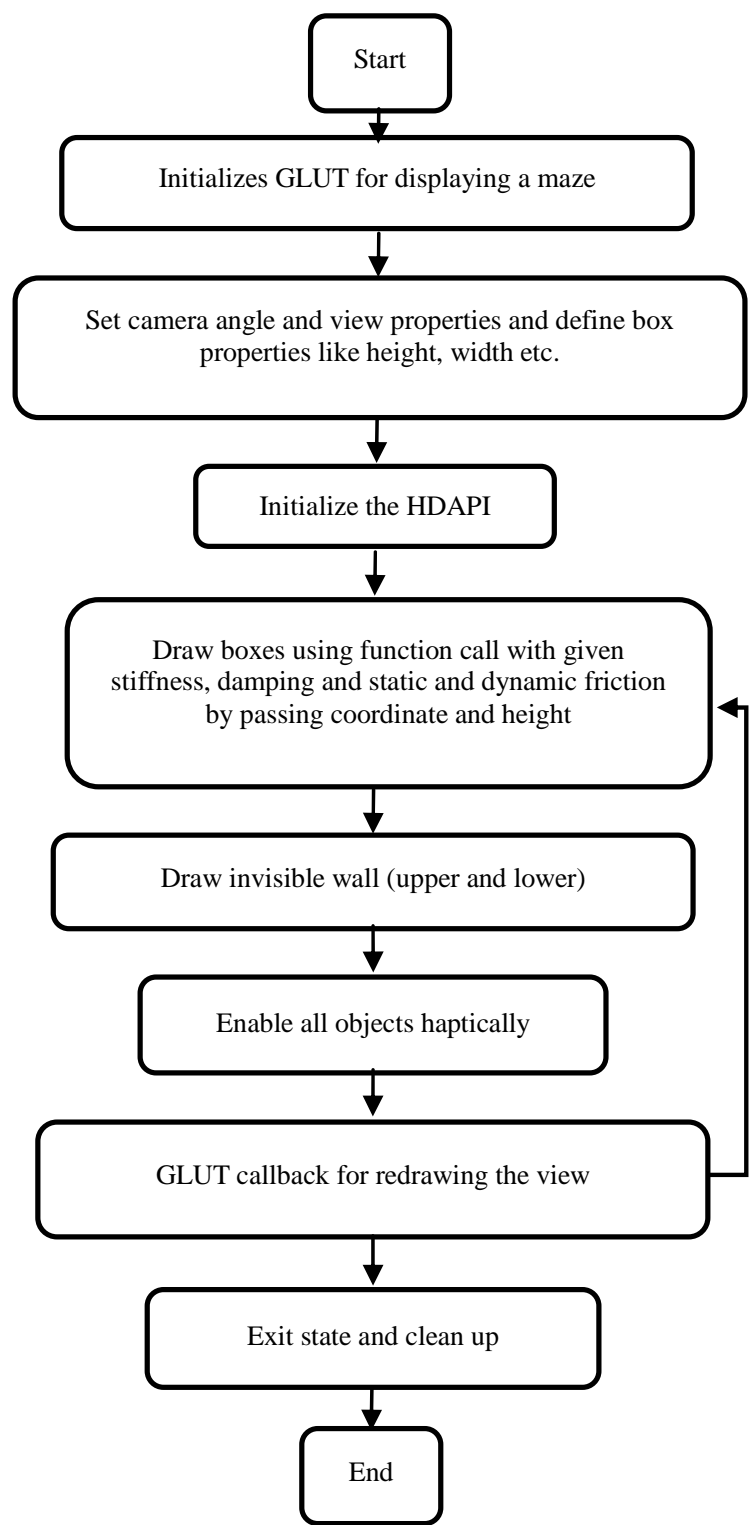

Figure 8. Flowchart used for creation of wall maze.

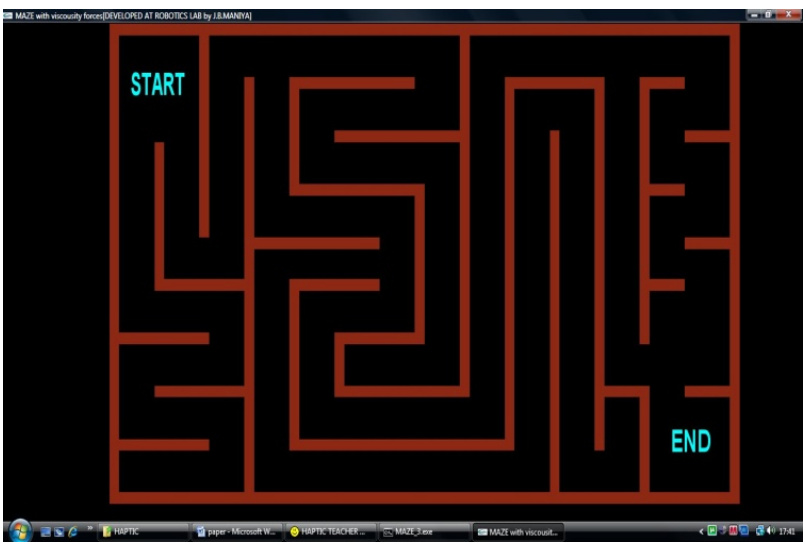

Figure 9. Wall exercise. 


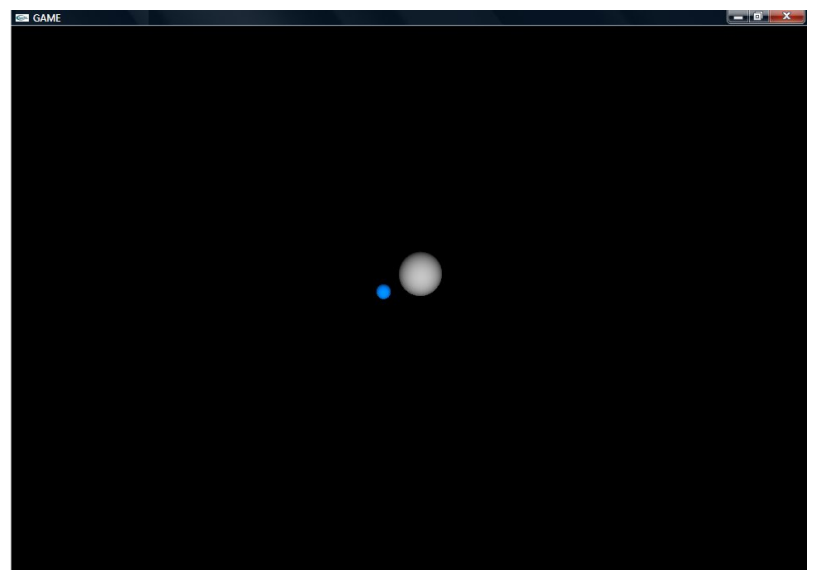

Figure 10. Game exercise.

to use it with new application. Figure 11 shows GUI for developed system.

\section{Experimental Validations on Subjects}

Experiments were conducted on four subjects of age group 11-14 years as subjects from this age group were readily available. In alphabet and number exercise, they were asked to move their hand along predefined path which create letters or number using wall scenario.

Figure 12 shows time taken by one subject to complete the different letters of the alphabet. This Figure shows results for introduction, training and testing. It is seen that when the test is introduced to subject, he takes more time as compared to during training and testing. This clearly shows the improvement in writing speed of the subject. After getting practice on individual letter, subjects were given exercise to write words. The average time taken to complete different words for different subjects is shown in Figure 13.

Here also we see considerable improvement in the performance of the subjects in terms of speed of writing text.

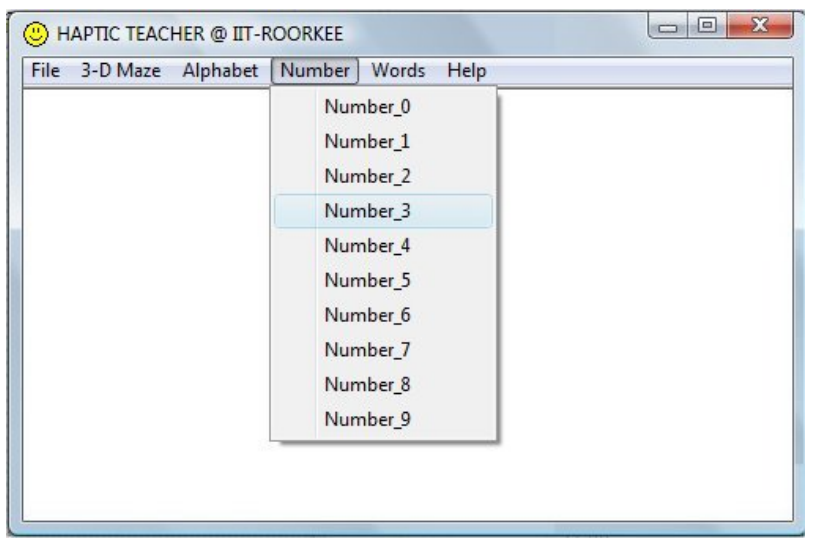

Figure 11. Graphic User Interface (GUI).

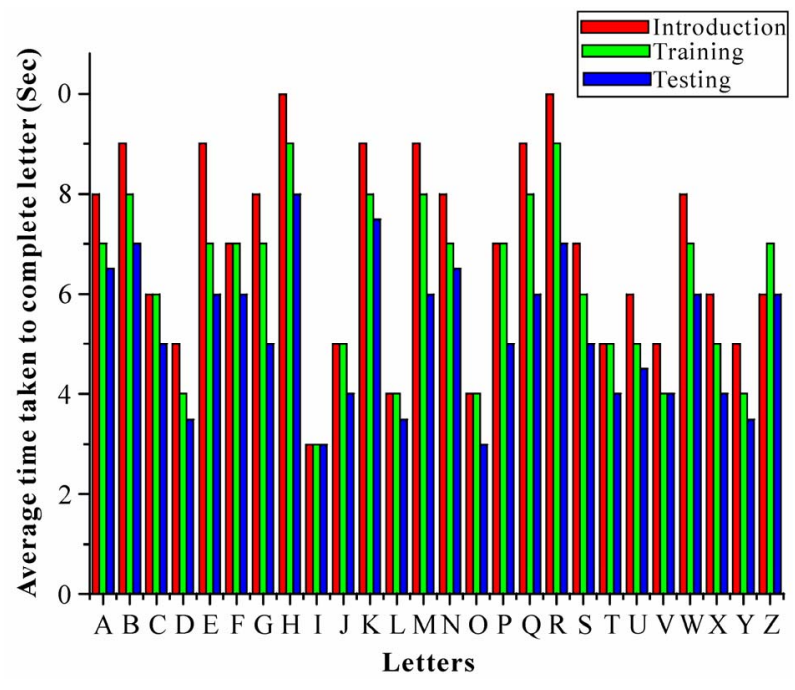

Figure 12. Time taken to complete different letters by one subject.

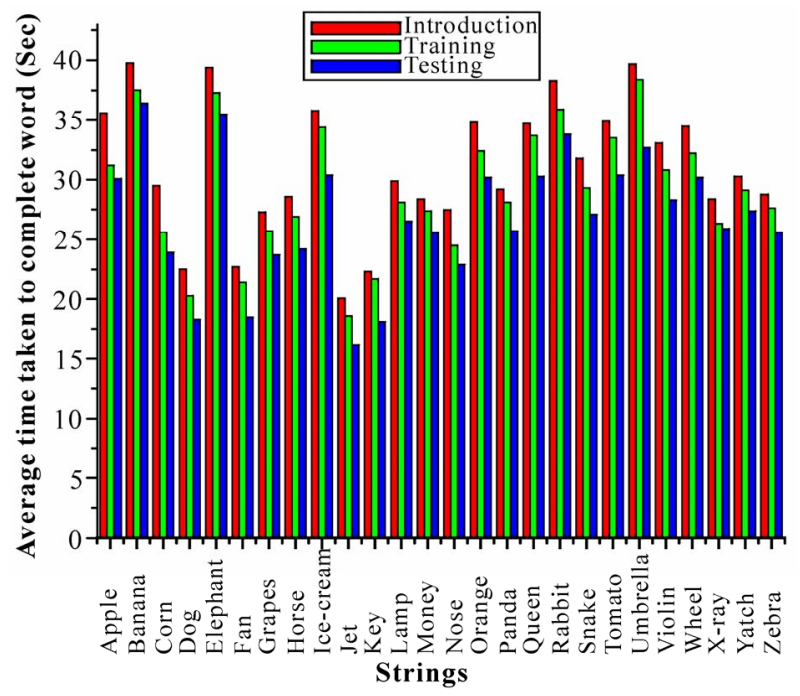

Figure 13. Average time taken by subjects to complete different words.

Figure 14 shows results for writing of numbers. Here also we see that subjects have shown improved performance in writing numbers after they got training on this system. In maze exercise, subjects had to take decision and move their hand through feasible path from many available paths to reach at end point from start point. Figure 15 shows the performance of subjects when they performed on wall and constrained maze.

We observe from this Figure that constrained maze requires comparatively less time as compared to wall maze to complete the task of traveling from start point to end point. Figure 16 shows performance of subjects after training on game object. We observe improvement in subject performance after getting training on designed 


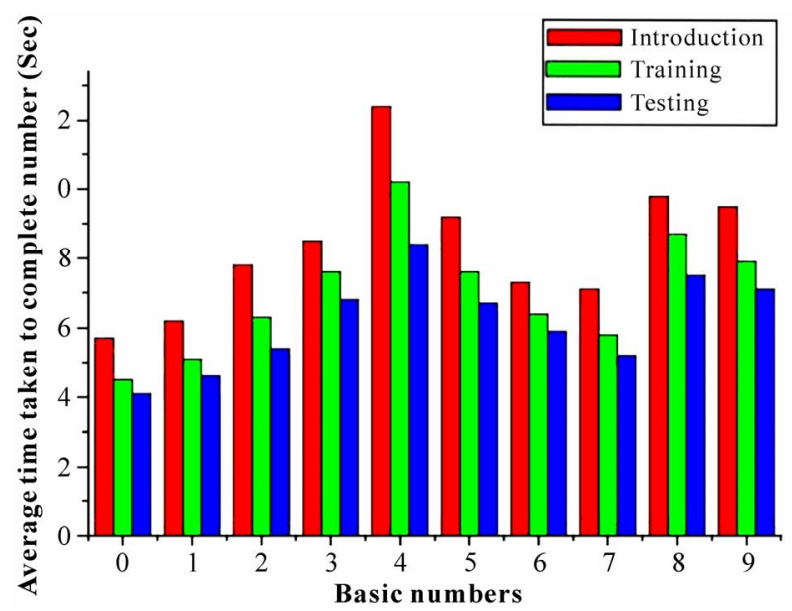

Figure 14. Average time taken by subjects to complete numbers.

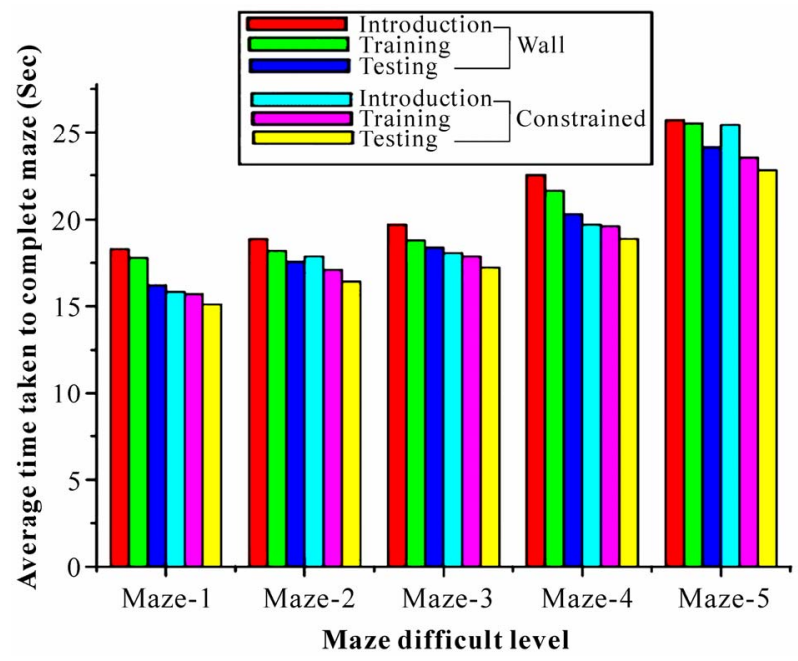

Figure 15. Average time taken by subjects to complete different maze level.

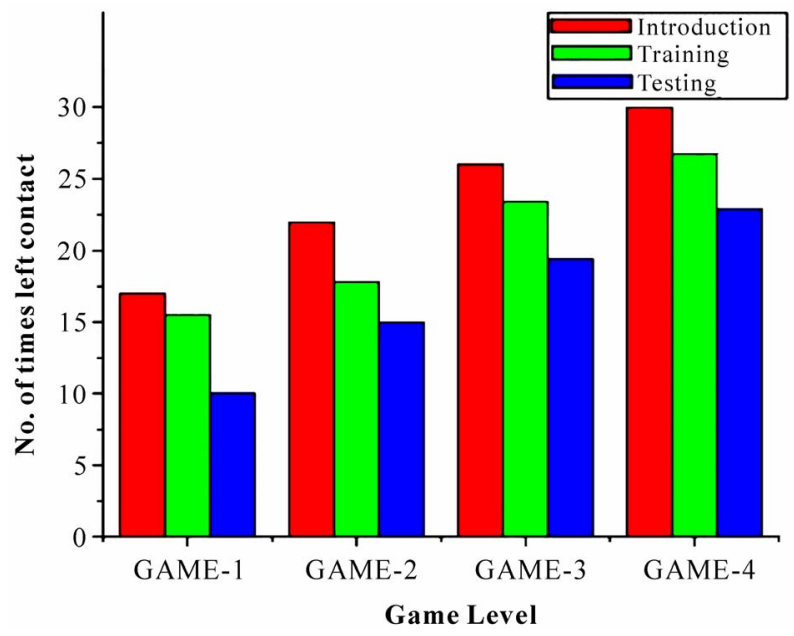

Figure 16. No. of times contact loss from ball. system of game. As they practice more on game scenario, they are less like to lose contact with randomly moving sphere on the screen.

\section{Conclusions}

This work presented the idea of use of virtual objects to improve the motor skill of the patients suffering from motor disorder. To validate the idea experiments were conducted on children aged 11-14 years. It is revealed that after being introduced to system, they adapt it rapidly and their skill improved and also time taken to accomplish the task has been reduced significantly. This system has potential to train motor disordered patients and stroke patients. The improvement in performance of the children in term of speed by which they created the object is encouraging. This system can be further improved by generalizing the strings and by incorporating full sentences. The game scenario also can be added which provides good exercise.

\section{REFERENCES}

[1] D. Grossi, G. Calise, C. Correra and L. Trojano, "Selective Drawing Disorders after Right Sub cortical Stroke: A Neuropsychological Premorbid and Follow-up Case Study," Italian Journal of Neurological Science, Vol. 17, No. 3, 1996, pp. 241-248.

[2] G. M. Prisco, C. A. Avizzano, M. Calcara, S. Ciancio, S. Pinna and M. Bergamasco, “A Virtual Environment with Haptic Feedback for the Treatment of Motor Dexterity Disabilities," International Conference on Robotics and Automation, Vol. 4, 1998, pp. 3721-3726.

[3] K. Henmi and T. Yoshikawa, "Virtual Lesson and Its Application to Virtual Calligraphy System,” IEEE International Conference on Robotics and Automation, Vol. 2, 1998, pp. 1275-1280.

[4] R. B. Gillespie, M. S. O’Modhrain, P. Tang, D. Zaretzky and C. Pham, "The Virtual Teacher," ASME International Mechanical Engineering Conference and Exposition, Anaheim, CA, 1998, pp. 3354-3361.

[5] N. Pemalete, W. Yu, R. Dubey and W. Moreno, "Development of a Robotic Haptic Interface to Assist the Performance of Vocational Tasks by People with Disabilities," IEEE International Conference on Robotics and Automation, Washington, DC, 2002, pp. 1269-1274,

[6] C. L. Teo, E. Burdet and H. P. Lim, “A Robotic Teacher of Chinese Handwriting,” 10th Symposium on Haptic Interfaces for Virtual Environment and Teleoperator Systems (HAPTICS.02), Orlando, 2002, pp. 335-341.

[7] D. Feygin, M. Keehner and F. Tendick, "Haptic Guidance: Experimental Evaluation of a Haptic Training Method for a Perceptual Motor Skill," 10th Symposium on Haptic Interfaces for Virtual Environment and Teleoperator Systems (HAPTICS.02), Orlando, 2002, pp. 40-47.

[8] N. Pernalete, S. Edwards, R. Gottipati, J. Tipple, V. 
Kolipakam and R. V. Dubey, "Eye-Hand Coordination Assessment/Therapy Using a Robotic Haptic Device," IEEE 9th International Conference on Rehabilitation Robotics, Chicago, IL, USA, 2005, pp. 25-28.

[9] J. Mullins, C. Mawson and S. Nahavandi, "Haptic Handwriting Aid for Training and Rehabilitation,” IEEE International Conference on Systems, Man and Cybernetics, USA, 2005, pp. 2690-2694.

[10] J. L. Grantner, R. Gottipati, N. Pernalete, G. A. Fodor and S. Edwards, "Intelligent Decision Support System for Eye-Hand Coordination Assessment," The 14th IEEE International Conference on Fuzzy Systems, USA, 2005, pp. 55-60.

[11] M. Mansour, M. Eid, and A. E. Saddik, "A Multimedia Handwriting Learning and Evaluation Tool," International Workshop on Educational Multimedia and Multimedia Education, Augsburg, Germany, 2007, pp. 103108.

[12] C. Gouy-Pailler, S. Zijp-Rouzier, S. Vidal and D. Chêne, "A Haptic Based Interface to Ease Visually Impaired Pupils' Inclusion in Geometry Lessons," Lecture Notes in Computer Science, Proceedings of the 4th International Conference on Universal Access in Human-Computer Interaction: Applications and Services, Beijing, China,
2007, pp. 598-606.

[13] R. Palluel-Germain, F. Bara, A. Hillairet de Boisferon, B. Hennion, P. Gouagout and E. Gentaz, "A Visuo-Haptic Device-Telemaque-Increases Kindergarten Children's Hand-writing Acquisition,” IEEE Second Joint EuroHaptics Conference and Symposium on Haptic Interfaces for Virtual Environment and Teleoperator Systems (WHC’07), Tsukaba, 2007, pp. 72-77.

[14] K. Rassmus-Grohn, C. Magnusson and H. Efiring, “AHEADAudio-Haptic Drawing Editor and Explorer for Education," HAVE 2007-IEEE International Workshop on Haptic Audio Visual Environments and Their Applications, Ottawa, Canada, 2007, pp. 62-66.

[15] R. Kayyali, S. Shirmohammadi, A. E. Saddik and E. Lemaire, "Daily-Life Exercises for Haptic Motor Rehabilitation," IEEE International Workshop on Haptic Audio Visual Environments and Their Applications, Ottawa, Canada, 2007, pp. 118-123.

[16] B. Bayart, A. Pocheville and A. Kheddar, “An Adaptive Haptic Guidance Software Module for I-TOUCH: Example Through a Handwriting Teaching Simulation and a 3D Maze," IEEE International Workshop on Haptic Audio Visual Environments and Their Applications, Canada, October, 2005, pp. 51-56. 